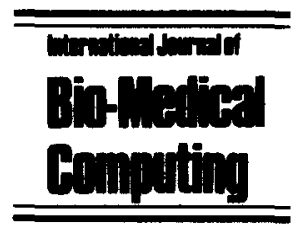

\title{
PC program for assessing the effect of a treatment when subjects are growing: the randomized parallel groups design
}

\author{
Charles J. Kowalski ${ }^{\mathrm{a}, \mathrm{b}}$, Emet D. Schneiderman* ${ }^{\mathrm{c}}$, Stephen M. Willis ${ }^{\mathrm{c}}$ \\ ${ }^{a}$ Department of Biologic and Materials Sciences, Dental School, b The Center for Statistical Consultation and Research, \\ The University of Michigan, Ann Arbor, MI 48109 USA

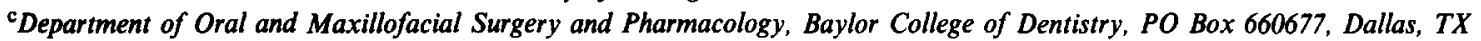 \\ 75266-0677, USA
}

Received 1 November 1993; accepted 1 December 1993

\begin{abstract}
A method for separating the effects of a treatment from those of normal growth and development in the case of a randomized parallel groups design with pre- and post-treatment measures is described and implemented. The program allows the user to enter either summary statistics (published data are often in this form), or the pre- and posttreatment measurements for each individual. The program is illustrated using data reflecting the extent to which a treatment can be expected to impede normal growth, but the method and program are more general than this. All that is required is that the measurement be one that normally increases over time.
\end{abstract}

Keywords: Randomized studies; Repeated measurements; Treatment effects; Growth; PC program

\section{Introduction}

The randomized parallel groups design with preand post-treatment measures is a simple, widely used study design in biomedical research (chapter 3 of Ref. [1]). In this design, subjects are randomly assigned to a treatment $(\mathrm{T})$ or a control $(\mathrm{C})$ group, and measured at comparable times before and some time after the treatment (TX) is administered. The data so collected are then analysed with a view towards comparing: (1) the pretreatment mean values in the groups (i.e., did the $\mathrm{T}$ and $\mathrm{C}$ groups start with comparable values?); (2)

\footnotetext{
* Corresponding author.
}

the pre- and post-measurements within each of the groups (i.e., did the $\mathrm{T}$ and/or $\mathrm{C}$ groups change from time 1 to time 2?); (3) the changes in the groups (i.e., did the $\mathrm{T}$ and $\mathrm{C}$ groups exhibit equal amounts of change?)

Hypothesis tests corresponding to these comparisons are well developed [2-4] and software for performing these tests is accessible to most researchers. Stanek [4] considered testing 1-3 in the context of repeated measures analysis of variance (RM ANOVA), analysis of covariance (ANCOVA), and seemingly unrelated regression (SUR) models. These are closely related and give identical estimates for certain of the parameters in the models; but the assumptions underlying the 
methods differ, and estimates of the variances of the parameters differ. The critical question in choosing the appropriate test is whether differences in pre-TX means are viewed as real or are considered to be the result of pure measurement error. If real, RM ANOVA appears to be the best choice (the relationship between RM ANOVA and simple $t$-tests in this context is given in Ref. [2]); if due to measurement error, either ANCOVA or SUR models should probably be employed, but the choice between these two is not clear-cut. SUR models can be expected to produce estimates with smaller variances, but this gain is somewhat compromised by lack of exact distributions for the test statistics. In any event, software for testing $1-3$ is readily available.

Less emphasis has been accorded the estimation of the effect of the TX relative to the control. In this paper we describe, illustrate, and make available a user-friendly, menu-driven PC program which can be used to estimate and compute confidence intervals for an index of this relative effectiveness in the situation in which the $T X$ is designed to affect (either to stimulate or suppress) a measurement subject to growth. This measurement is made in both the $\mathrm{T}$ and $\mathrm{C}$ groups before and after the application of the TX. The theory behind our method was developed in Ref. [1] (p. 210) in the somewhat different - but related context of describing the TX effects for 'deteriorating conditions', i.e. the response variable is one for which high values are 'bad' and the measurement is assumed to be non-decreasing. An example given in Ref. [1] is that of the DMFS score (the number of decayed, missing and filled surfaces of a patient's permanent teeth), which cannot decrease with the passage of time and, in the absence of TX, can be expected to increase. We will illustrate and discuss the method in the context of growth, but follow the notation and computational procedures detailed in Ref. [1]. This will allow the interested reader to relate our discussion to other material given in Ref. [1], and provides a source for additional examples.

Let $\Delta_{1}$ and $\Delta_{2}$ denote the underlying mean changes in the response variable from baseline. We use the subscript 1 to denote the $T$ group, and 2 for the control. By assumption, neither $\Delta_{1}$ nor $\Delta_{2}$ can be negative. The quantity

$P=\frac{\Delta_{2}-\Delta_{1}}{\Delta_{2}}$

is, in the context of a deteriorating condition, then the proportion by which the mean worsening associated with the control group has been diminished by the TX. Values of $\boldsymbol{P}$ close to zero show a relatively small TX effect; values near 1 , a large TX effect. Dubey [5] developed a somewhat different method for confidence interval construction which allows $\Delta_{1}$ to be negative, so that $P$ can be larger than 1 . In the context of deteriorating conditions, this corresponds to a TX which is positively curative, i.e., one that has reversed the course of the disease. We follow Fleiss's development [1] since our primary interest is in growth, and $\Delta_{1}<0$ would correspond to 'shrinkage'.

In the context of growth, $P$ is the proportion by which mean normal growth has been slowed by TX. Values of $P$ near $0\left(\Delta_{1}\right.$ near $\left.\Delta_{2}\right)$ indicate that the TX has been ineffective. Values near $1\left(\Delta_{1}\right.$ near 0 ) indicate that the TX has suppressed growth. Values less than zero $\left(\Delta_{1}>\Delta_{2}\right)$ indicate that the TX has stimulated growth (a negative reduction is a gain).

Let $\bar{D}_{1}$ and $\bar{D}_{2}$ denote the two sample mean changes calculated from samples of sizes $n_{1}$ and $n_{2}$. In the notation of Ref. [1],

$\bar{D}_{i}=\frac{1}{n_{j}} \sum_{j=1}^{n_{j}}\left(X_{i j}-Z_{i j}\right)=\bar{X}_{i}-\bar{Z}_{i}$

i.e. the baseline measurements are denoted by $Z$; the post-TX measurements by $X$. For example, $X_{i j}$ is the post-TX measurement for the $j$ th individual $\left(j=1,2, \ldots, n_{i}\right)$ in group $i(i=1,2)$.

We consider two cases. In the first, the simple, unadjusted means (Eq. 2) are used to estimate $P$. In the second, we use instead so-called covarianceadjusted means, which are derived through use of ANCOVA. These adjust the $\bar{D}_{i}$ values for any imbalances in the response variable that might have existed prior to TX. In randomized studies, we expect the groups will be comparable at baseline, but this expectation is not always realized in practice. 


\section{Unadjusted means}

In this case we use the simple mean differences as given in Eq. 2. $P$ can then be estimated by

$$
\hat{P}=\frac{\bar{D}_{2}-\bar{D}_{1}}{\bar{D}_{2}}
$$

and confidence intervals for $P$ can be constructed by first using Fieller's theorem (Ref. [1], p. 42) to get a confidence interval for $R=\Delta_{1} / \Delta_{2}$, then using the fact that $P=1-R$ to derive the corresponding interval for $P$. To get a $(1-\alpha) \times 100 \%$ confidence interval, first compute

$g=\frac{t_{1-\alpha / 2}^{2}\left(n_{1}+n_{2}-2\right) s_{\mathrm{D}}^{2}}{n_{2} \bar{D}_{2}^{2}}$

where $t_{1-\alpha / 2}\left(n_{1}+n_{2}-2\right)$ is the $(1-\alpha / 2)$ th percentile of the $t$ distribution with $n_{1}+n_{2}-2$ degrees of freedom, and $s_{\mathrm{D}}^{2}$ is the pooled estimate of the variance of the differences in the two groups, namely,

$s_{\mathrm{D}}^{2}=\frac{\left(n_{1}-1\right) s_{\mathrm{D} 1}^{2}+\left(n_{2}-1\right) s_{\mathrm{D} 2}^{2}}{n_{1}+n_{2}-2}$

where $s_{\mathrm{D} 1}^{2}$ and $s_{\mathrm{D} 2}^{2}$ are the variances of the differences in the two groups. Confidence limits for $R$ have the lower limit

$$
\begin{aligned}
R_{\mathrm{L}}= & \frac{1}{1-g}\left[\hat{R}-\frac{t_{1-\alpha / 2}\left(n_{1}+n_{2}-2\right) s_{\mathrm{D}}}{\bar{D}_{2}}\right. \\
& \sqrt{\left.\frac{1-g}{n_{1}}+\frac{\hat{R}^{2}}{n_{2}}\right]}
\end{aligned}
$$

and upper limit

$$
\begin{aligned}
R_{\mathrm{U}}= & \frac{1}{1-g}\left[\hat{R}-\frac{t_{1-\alpha / 2}\left(n_{1}+n_{2}-2\right) s_{\mathrm{D}}}{\bar{D}_{2}}\right. \\
& \sqrt{\left.\frac{1-g}{n_{1}}+\frac{\hat{R}^{2}}{n_{2}}\right]}
\end{aligned}
$$

The confidence limits for $P$ are then $\left(1-R_{\mathrm{U}}\right.$, $1-R_{\mathrm{L}}$ ).

\section{Covariance-adjusted mean differences}

The above computations are based on the raw, unadjusted differences $D=X-Z$ in the two groups. As indicated in Ref. [1] (p. 213), it is possible that covariance-adjusted differences will produce narrower confidence intervals should the groups differ at baseline. The adjusted differences are based on the ANCOVA model (Ref. [1] p. 194):

$X_{i j}=\mu_{i}+\beta\left(Z_{i j}-\bar{Z}\right)+e_{i j}$

where $e_{i j}$ is a normally distributed error term with mean 0 and variance $\sigma^{2}$. We write $e_{i j} \sim N\left(0, \sigma^{2}\right)$. The least squares estimators of the parameters of the model are

$$
\begin{aligned}
\hat{\beta} & =\frac{\sum_{i=1}^{2} \sum_{j=1}^{n_{i}}\left(X_{i j}-\bar{X}_{i}\right)\left(Z_{i j}-\bar{Z}_{i}\right)}{\sum_{i=1}^{2} \sum_{j=1}^{n_{i}}\left(Z_{i j}-\bar{Z}_{i}\right)^{2}} \\
& =\frac{W S P(X Z)}{\operatorname{WSS}(Z)}
\end{aligned}
$$

and

$\hat{\mu}_{i}=\bar{X}_{i}-\hat{\beta}\left(\bar{Z}_{i}-\bar{Z}\right)$

where $\bar{Z}$ is the overall (weighted) mean of the $Z_{i j}$. In Ref. [9] we introduce the WSS and WSP notation used in the ANCOVA tables of Ref. [1]. These are read 'within sum of squares' and 'within sum of products', respectively. In terms of simpler, more familiar quantities we note that

$W S S(Z)=\left(n_{1}-1\right) s_{1 Z}^{2}+\left(n_{2}-1\right) s_{2 Z}^{2}$ 
and

$$
\begin{aligned}
& W S P(X Z)=\left(n_{1}-1\right) s_{1 X} s_{1 Z} r_{1}(X, Z) \\
& +\left(n_{2}-1\right) s_{2 X} s_{2 Z} r_{2}(X, Z) \\
& =\left(n_{1}-1\right) \operatorname{Cov}_{1}(X, Z)+\left(n_{2}-1\right) \operatorname{Cov}_{2}(X, Z)
\end{aligned}
$$

where, for example, $s_{1 X}$ (respectively, $s_{1 X}^{2}$ ) is the standard deviation (respectively, variance) of $X$ in group 1; and $\operatorname{Cov}_{1}(X, Z)$ (respectively, $r_{1}(X, Z)$ ) is the covariance (respectively, correlation) between $X$ and $Z$ in group 1 . Below, and in the output of the program, we use S.D. to stand for standard deviation.

The covariance-adjusted means within the two groups are then

$\bar{D}_{1}^{\prime}=\bar{D}_{1}-\hat{\beta}\left(\bar{Z}_{1}-\bar{Z}\right)$

and

$$
\bar{D}_{2}^{\prime}=\bar{D}_{2}-\hat{\beta}\left(\bar{Z}_{2}-\bar{Z}\right)
$$

We then compute

$$
\hat{R}^{\prime}=\frac{\bar{D}_{1}^{\prime}}{\bar{D}_{2}^{\prime}}
$$

Now, the form of Fieller's theorem used above cannot be applied here since the adjusted estimators are dependent (they both involve $\hat{\beta}$ and $\bar{Z})$. Instead, we compute

$g=\frac{t_{1-\alpha / 2}^{2}\left(n_{1}+n_{2}-3\right) s_{D^{\prime}}^{2} a_{2}^{2}}{\left(\bar{D}_{2}^{\prime}\right)^{2}}$

In Eq. 16, $t$ refers to the $t$-distribution as before (one degree of freedom fewer since we estimated $\beta)$. We also need

$$
s_{D^{\prime}}^{2}=\operatorname{WSS}\left(D^{\prime}\right) /\left(n_{1}+n_{2}-3\right)
$$

where

$$
W S S\left(D^{\prime}\right)=W S S(D)-\frac{[W S P(D Z)]^{2}}{W S S(Z)}
$$

Again, as in Eqs. 11 and 12, it is possible to express the quantities in Eq. 18 in terms of simple summary statistics. We have, for example, $W S S(D)=$ $\left(n_{1}-1\right) s_{1 D}^{2}+\left(n_{2}-1\right) s_{2 D}^{2}$. We follow Fleiss [1] so that the reader will have the opportunity to relate our discussion to this more extended material.

In Eq. 16, and subsequently, we also need to define

$a_{i}^{2}=\frac{1}{n_{i}}+\frac{\left(\bar{Z}_{i}-\bar{Z}\right)^{2}}{W S S(Z)}$

and

$a_{12}=\frac{\left(\bar{Z}_{1}-\bar{Z}\right)\left(\bar{Z}_{2}-\bar{Z}\right)}{\operatorname{WSS}(Z)}$

Finally, we let

$$
Q=(1-g) a_{1}^{2}+\left(\hat{R}^{\prime}\right)^{2} a_{2}^{2}-2 \hat{R}^{\prime} a_{12}+g \frac{a_{12}^{2}}{a_{2}^{2}}
$$

Then the $(1-\alpha) \times 100 \%$ confidence interval for $R^{\prime}$ is given by

$$
\begin{aligned}
& \frac{1}{1-g}\left[\left(\hat{R}^{\prime}-g \frac{a_{12}}{a_{2}^{2}}\right)\right. \\
& \pm \frac{t_{1-\alpha / 2}^{2}\left(n_{1}+n_{2}-3\right) s_{D}^{2}, \sqrt{Q}}{\bar{D}_{2}^{\prime}}
\end{aligned}
$$

For reasons given in section 6 below, we also compute and print the estimated correlation between $D$ and $Z$, namely,

$r(D, Z)=\frac{W S P(D Z)}{\sqrt{W S S(D) W S S(Z)}}$ 
This is the 'pooled within-group' correlation coefficient, which combines data on the relationship between $D$ and $Z$ from both groups. It is not, as is sometimes thought, found by computing the correlation coefficient from the data from both groups considered as a single group (see also, Ref. [6], p. 147).

\section{The program}

The program is invoked by issuing the command gsruni txrpg. The user is first asked whether the data are (i) in a (ASCII or GAUSS) file, or if (ii) summary statistics are to be entered interactively. If (i), the user is prompted for the type, location, and name of the file, which is assumed to be of the following structure: group indicator in column 1, pre- and post-measurements in columns 2 and 3. The user is next asked for the confidence coefficient (e.g., 0.95) to be used to construct the confidence interval. Color coded plots of the 'growth curves' of the individuals comprising the two groups are shown; descriptive statistics are given; estimates of the model parameters and the covariance adjusted mean differences are printed; and the values of the unadjusted and adjusted estimators of $P$ and their corresponding confidence intervals are provided.

If the user responds (ii), they are prompted for the values of the summary statistics necessary to estimate $P$. These may be in the form of pre- and post-TX values or pre-TX values and differences, i.e., the user can enter either $Z, X$ statistics or $Z, D$ statistics. The information we require for the case of $Z, X$ statistics is summarized in Table 1 .

If the user indicates that they have $Z, D$ summary statistics, the prompts are similar: The only change is from 'Post-TX' to 'Differences'. The option of providing summary statistics should prove useful since the results of studies of the type considered here are often published in one or another of these forms. See Ref. [1], p. 212 for an example of $Z, D$ data; Ref. [6], p. 237 for $Z, X$ data.

\section{Some examples}

Our first example is based on a subset of the data first considered by Box [7]. For convenience,
Table 1

Information required if data being inputted is $Z, X$ statistics

Number in TX group

Pre-TX mean in TX group

Pre-TX S.D. in TX group

Post-TX mean in TX group

Post-TX S.D. in TX group

Pre-post correlation in TX group

Number in control group

Pre-TX mean in control group

Pre-TX S.D. in control group

Post-TX mean in control group

Post-TX S.D. in control group

Pre-post correlation in control group

these are reproduced in Table 2 in the form of an ASCII data file which will be accepted by our program.

The first column is the group indicator $(1=\mathrm{TX}$, $2=$ Control). The next two columns contain the pre- and post-TX measurements. The tabulated values are the weights $(\mathrm{g})$ of 20 rats. The TX involved the use of the drug thiouracil, which was added to the drinking water of the rats in this

Table 2

Subset of data, first used by Box [7], used in this experiment

\begin{tabular}{lll}
\hline Group & Pre-TX & Post-TX \\
\hline 1 & 61 & 86 \\
1 & 59 & 80 \\
1 & 53 & 79 \\
1 & 59 & 88 \\
1 & 51 & 75 \\
1 & 51 & 75 \\
1 & 56 & 78 \\
1 & 58 & 69 \\
1 & 46 & 61 \\
1 & 53 & 72 \\
2 & 57 & 86 \\
2 & 60 & 93 \\
2 & 52 & 77 \\
2 & 49 & 67 \\
2 & 56 & 81 \\
2 & 46 & 70 \\
2 & 51 & 71 \\
2 & 63 & 91 \\
2 & 49 & 67 \\
2 & 57 & 82 \\
\hline
\end{tabular}


group. Using more extensive longitudinal data (five time points), Box showed clear differences between the growth curves in these groups. In the above, we use only the first time point posttreatment, so we are considering only the immediate impact of the TX.

Color coded plots of the 'growth curves' of the individuals comprising the two groups are plotted as shown in Fig. 1. This was called a 'tilted line segment plot' by McNeil [8]. It gives an indication of the overall course of growth, inter-individual variability in growth, some insight into tracking behavior [12-16], and may be useful in identifying outliers.

Descriptive statistics and estimates of the model parameters are provided, as well as the values of the unadjusted and adjusted estimators of $P$ and their corresponding confidence intervals. The descriptive statistics for the above data are given in the form shown in Table 3.

Both estimates of PHAT, the proportion reduction in growth due to the treatment, are positive, indicating that the thiouracil has retarded natural growth, but not to any great extent. The fact that zero is contained in the $95 \%$ confidence intervals means that the amount of reduction is not significantly different from zero at the $5 \%$ level of significance.

The user may only have access to published summary statistics as given, for example, in Table 4 (taken from Ref. [6], p. 237). These data represent pre- and post-measurements of the 'PreSchool Inventory', which measures the general knowledge of pre-school-age children. The control group includes children from ordinary Head Start programs; the TX group children who received an

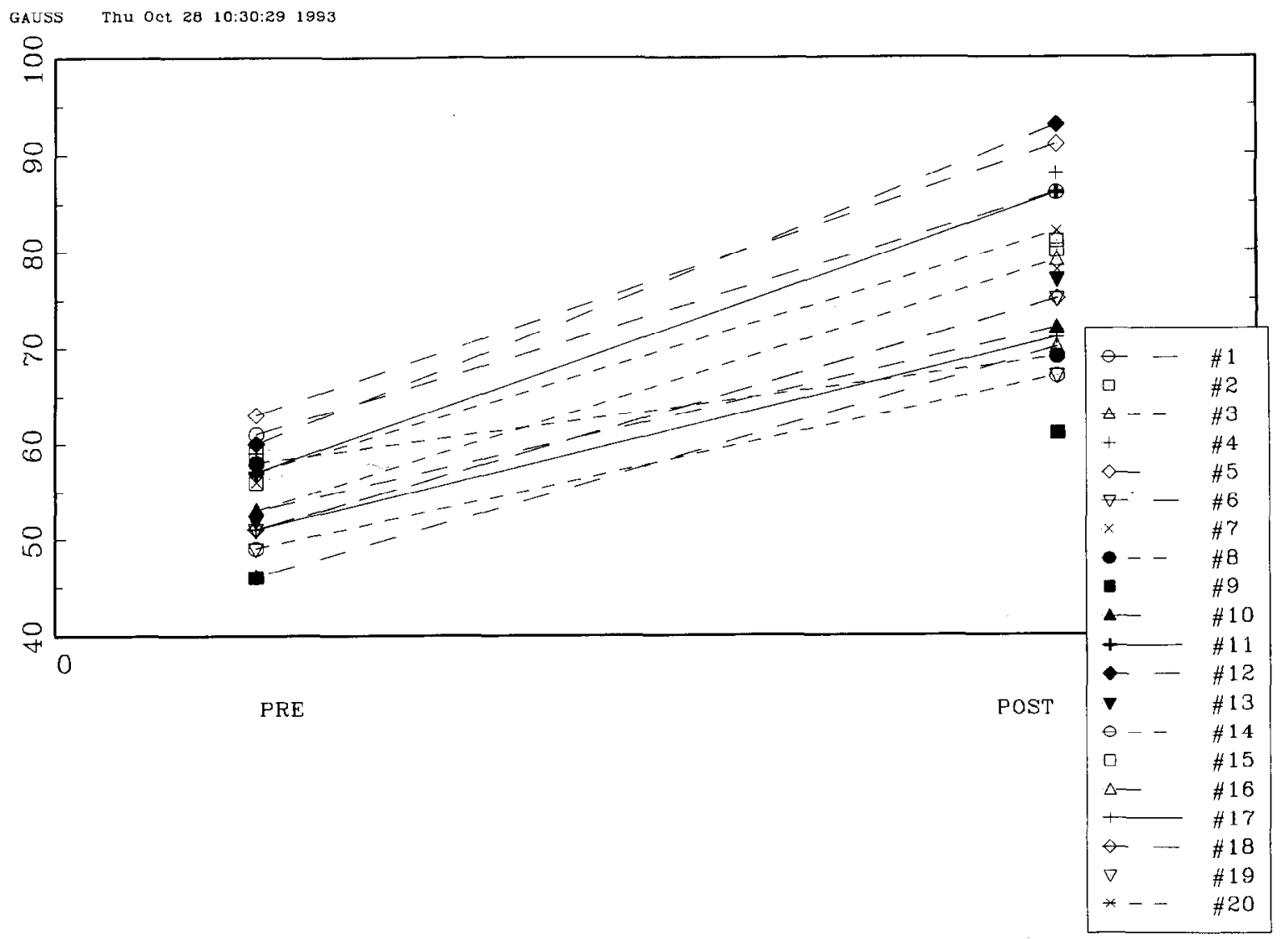

Fig. 1. Tilted line segment plot of the pre- and post-measurements in the two groups. 
Table 3

Descriptive statistics for the data in Table 2



innovative curriculum. After indicating that summary statistics are to be entered (in $Z, X$ form), the user is prompted for these values. The output consists of the descriptive statistics and estimates of the model parameters as above; and estimates of the unadjusted and adjusted estimates of $P$ and the corresponding confidence intervals. When only summary statistics are available the plot consists of two lines, one for each group, showing the amount of mean change by connecting the premeasure mean to the postmeasure mean. For the data in Table 4 we get the output presented in Table 5.

In this case, the proportion reduction in 'growth' is negative, indicating that the innovative curriculum has significantly increased the PreSchool Inventory mean score. (Again, the reader is reminded that a negative reduction represents a
Table 4

Summary statistics, taken from p. 237 of Ref. [6]

\begin{tabular}{llll}
\hline & Pre & Post & Corr \\
\hline $\mathrm{TX}(\mathrm{N}=157)$ & Mean $=17.1$ & Mean $=23.3$ & $r=0.67$ \\
& S.D. $=6.1$ & S.D. $=4.6$ & \\
$\mathrm{C}(\mathrm{N}=669)$ & Mean $=14.6$ & Mean $=18.9$ & $r=0.78$ \\
& S.D. $=6.2$ & S.D. $=5.8$ & \\
\hline
\end{tabular}

gain.) This increase is significant (at the $5 \%$ level of significance) since the $95 \%$ confidence interval for PHAT does not contain zero.

The output which appears on the screen is automatically saved in a file called TXRPG.OUT which can be read into a word processor, edited, annotated, and printed.

This program is a part of a series of programs for accomplishing various kinds of longitudinal data analyses. These include programs for estimating the average growth curve (AGC) in a group of subjects [9-11]; for measuring tracking behavior [12-16]; for growth prediction [17-19]; and for comparing the AGCs in several groups [20,21]. Details on obtaining copies of these programs, hardware requirements, etc., are given in Appendix 1.

Table 5

Output for the data presented in Table 4

Pooled correlation between $D$ and $Z=-0.4688$

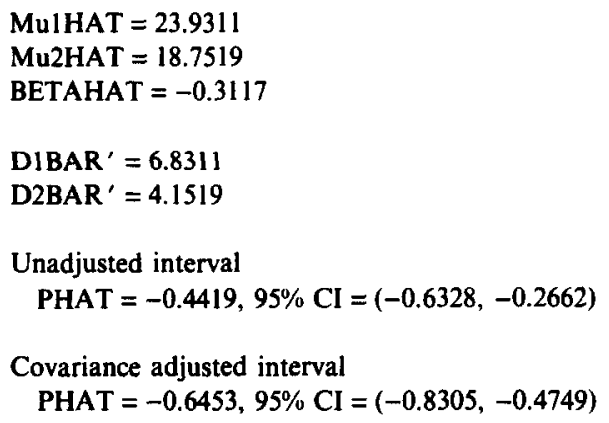




\section{Discussion}

We have described a program which can be used to estimate the extent to which a TX has altered the course of normal growth and development. It can also be used when the response variable represents a 'deteriorating condition', as described above. The object of inference is $P=\left(\Delta_{2}-\Delta_{1}\right) /$ $\Delta_{2}$, and the differences, $\Delta$, may be estimated as simple mean differences or by covariance-adjusted differences. The confidence intervals for covariance-adjusted estimators will generally be narrower, but there will probably be little difference unless the absolute value of the pooled withingroup correlation between $Z$ and $D$ exceeds 0.40 (Ref. [1], p. 215). Another interpretation of the pooled correlation between $Z$ and $D$, say $r_{P}(Z, D)$, can be given in terms of the efficiency of the analysis of covariance with $Z$ as the covariate relative to the corresponding $t$-test (in which $Z$ is ignored) for comparing the two groups. The relative efficiency is $R E=100 /\left[1-r_{P}^{2}(Z, D)\right]$ and it is seen that the higher the absolute value of $r_{P}(Z, D)$, the more important it is to account for it by using ANCOVA.

Input data can be in the form of individual measurements - group indicator in column 1, premeasures in column 2, postmeasures in column 3 ; or in the form of summary statistics (means, S.D.s and correlations in terms of either $Z$ and $X$, or $Z$ and $D$ ). This latter possibility is included to accommodate users who may have access only to this summary information. In some cases, the user may have to do some preprocessing to get the data into the form required by our program. For example, in Ref. [1] (p. 212) data are given in the form shown in Table 6. In this case, the user will have to convert the given covariances to correlations.

Table 6

Form in which the data is given in Fleiss (Ref. [1], p. 212)

\begin{tabular}{clll}
\hline & Pre & Diff & Covariance \\
\hline $\mathrm{TX}(\mathrm{N}=226)$ & Mean $=6.28$ & Mean $=2.97$ & 16.4817 \\
& S.D. $=7.77$ & S.D. $=4.41$ & \\
$\mathrm{C}(\mathrm{N}=225)$ & Mean $=7.50$ & Mean $=3.24$ & 7.7622 \\
$\vdots$ & S.D. $=8.23$ & S.D. $=4.26$ & \\
\hline
\end{tabular}

Table 7

Results produced when summary data from Fleiss (Ref. [1], p. 214) are entered

Pooled correlation between $D$ and $Z=0.3494$

MulHAT $=9.3625$

Mu2HAT $=10.6243$

BETAHAT $=0.1893$

D1BAR' $=3.0852$

$\mathrm{D} \mathrm{BAR}^{\prime}=3.1243$

Unadjusted interval

PHAT $=0.0833,95 \% \mathrm{CI}=(-0.1889,0.2974)$

Covariance adjusted interval

PHAT $=0.0125,95 \% \mathrm{CI}=(-0.2626,0.2281)$

The relationship to use is

$r(Z, D)=\operatorname{Cov}(Z, D) / s_{Z} s_{D}$

In the above example (Ref. [1], p. 214), for the TX group, $r_{1}(Z, D)=16.4817 /(7.77 * 4.41)=0.48$ 1. This can also be done for the $\mathrm{C}$ group $\left(r_{2}(Z\right.$, $D)=0.221$ ), and when these summary figures are entered into the program, the results are as shown in Table 7. In this example, the TX is one designed to slow the normal course of dental decay. Both estimates of $P$ are positive indicating that the TX has had a positive effect (in that the decay rate has decreased), but the effect is seen to be small ( $8 \%$ unadjusted; $1 \%$ adjusted). The fact that zero is included in both intervals is consistent with the fact that the difference between the $\bar{D}_{S}$ is not significantly different from zero. In this example, the widths of the unadjusted and adjusted intervals are essentially the same, so that 'covariance adjustment was not worth the effort with this set of data' (Ref. [1], p. 215). The width of the adjusted interval can be substantially less (see, for example, Ref. [22]), but, as noted earlier, only when the pooled correlation between $Z$ and $D$ exceeds 0.40 in absolute-value.

\section{Acknowledgement}

This work was supported by DE 08730 from the National Institute for Dental Research. 


\section{Appendix}

A full set of PC programs for longitudinal data analysis, including this program, can be obtained on 5.25 " or $3.5^{\prime \prime}$ diskettes (please request type) by sending US\$25 to defray the cost of handling and licensing fees. These programs require an 80386 or 80486 based personal computer (PC) running the MS-DOS operating system (version 5.0 or higher is recommended, although versions as low as $\mathbf{3 . 3}$ will suffice). 80386 computers must also be equipped with a 80387 math coprocessor. At least $4 \mathrm{Mb}$ of memory is required, and must be available to GAUSS386i, i.e., not in use by memory resident programs such as Windows. EGA or VGA graphic capabilities are required to display the color graphics; VGA or SVGA is suggested for optimally displaying the graphic results. Runtime modules are supplied with the programs so that no additional software (i.e., compiler or interpreter) is required to run these programs. One can create and edit ASCII data sets for use by these programs using the full screen editor supplied with MS-DOS version 5.0. The programs are written and compiled using GAUSS386i, version 3.0, require no additional installation or modification, and are run with a single command. When requesting the programs, address inquiries to the corresponding author and make checks payable to Baylor College of Dentistry.

\section{References}

[1] Fleiss JL: The Design and Analysis of Clinical Experiments, Wiley, New York, 1986.

[2] Brogan DR and Kutner MH: Comparative analyses of pretest-posttest research designs, Am Stat, 34 (1980) 229-232.

[3] Laird N: Further comparative analyses of pretestposttest research designs, Am Stat, 37 (1983) 329-333.

[4] Stanek EJ III: Choosing a pretest-posttest analysis, $\mathrm{Am}$ Stat, 42 (1988) 178-183.

[5] Dubey S.D.: On the determination of confidence limits for an index, Biometrics, 22 (1966) 603-609.

[6] Anderson S, Auquier A, Hauck WW, Oakes D, Vandaele W and Weisberg HI: Statistical Methods for Comparative Studies: Techniques for Bias Reduction, Wiley, New York, 1980.
[7] Box GEP: Problems in the analysis of growth and wear curves, Biometrics, 6 (1950) 362-389.

[8] McNeil D: On graphing paired data, Am Stat, 46 (1992) 307-311.

[9] Schneiderman ED and Kowalski CJ: Implementation of Hills' growth curve analysis for unequal-time intervals using GAUSS, Am J Hum Biol, 1 (1989) 31-42.

[10] Ten Have TR, Kowalski CJ and Schneiderman ED: PC program for analyzing one sample longitudinal data sets which satisfy the two-stage polynomial growth curve model, Am J Hum Biol, 3 (1991) 269-279.

[11] Schneiderman ED, Willis SM, Ten Have TR and Kowalski CJ: Rao's polynomial growth curve model for unequal-time intervals: a menu-driven GAUSS program, Int J Biomed Comput, 29 (1991) 235-244.

[12] Schneiderman ED, Kowalski CJ and Ten Have TR: A GAUSS program for computing an index of tracking from longitudinal observations, Am J Hum Biol, 2 (1990) 475-490.

[13] Schneiderman ED, Willis SM, Kowalski CJ and Ten Have TR: PC program for comparing tracking indices in several independent groups, Am J Ilum Biol, 4 (1992) 399-401.

[14] Schneiderman ED, Kowalski CJ, Ten Have TR and Willis SM: Computation of Foulkes and Davis' nonparametric tracking index using GAUSS, Am $J$ Hum Biol, 4 (1992) 417-420.

[15] Schneiderman ED, Willis SM, Kowalski CJ and Ten Have TR: A GAUSS program for computing the Foulkes-Davis tracking index for polynomial growth curves, Int J Biomed Comput, 32 (1993) 35-43.

[16] Kowalski CJ and Schneiderman ED: Tracking: concepts, methods and tools, Int. J. Anthropol, 7 (1992) 33-50.

[17] Schneiderman ED, Willis SM, Kowalski CJ and Ten Have TR: A PC program for growth prediction in the context of Kao's polynomial growth curve model, Comput Biol Med, 22 (1992) 181-188.

[18] Schneiderman ED, Willis SM, Kowalski CJ and Ten Have TR: Longer-term growth prediction using GAUSS, Comput Biol Med, 23 (1993) 149-154.

[19] Schneiderman ED, Kowalski CJ and Willis SM: Regression imputation of missing values in longitudinal data sets, Int J Biomed Comput, 32 (1993) 121-131.

[20] Ten Have TR, Kowalski CJ, Schneiderman ED and Willis SM: A PC program for performing multigroup longitudinal comparisons using the Potthoff-Roy analysis and orthogonal polynomials, Int $J$ Biomed Comput, 30 (1992) $103-112$.

[21] Ten Have TR, Kowalski CJ, Schneiderman ED and Willis SM: Two programs for performing multigroup longitudinal data analyses, Am J Phys Anthropol, 88 (1992) 25l-254.

[22] Wallenstein S, Fleiss JL and Chilton NW: Confidence intervals for percentage reduction in caries increments, $J$ Dent Res, 61 (1982) 828-830. 